\title{
Effects of Corneal Crosslinking for Keratoconus Assessed with Anterior Segment Optical Coherence Tomography
}

Fernando Fuentes Bonthoux ${ }^{1}$, Jeremías G. Galletti ${ }^{2}$, Adriana Tytiun ${ }^{3}$, Pablo A. Chiaradía ${ }^{3}$, Tomás Pförtner 4

1. ECOS (Clinical Ocular Studies) Laboratory, Buenos Aires, Argentina; Ophthalmology Division, Hospital de Clínicas José de San Martín, University of Buenos Aires, Argentina 2. Institute of Experimental Medicine, National Academy of Medicine/CONICET, Buenos Aires, Argentina 3. Ophthalmology Division, Hospital de Clínicas José de San Martín, University of Buenos Aires, Argentina 4. ECOS (Clinical Ocular Studies) Laboratory, Buenos Aires, Argentina

$\square$ Corresponding author: Jeremías G. Galletti, jeremiasg@gmail.com

Disclosures can be found in Additional Information at the end of the article

\section{Abstract}

Purpose: To report anterior segment optical coherence tomography (AS-OCT) findings in corneas of keratoconus patients treated with riboflavin-ultraviolet-A-light-induced crosslinking (CXL).

Methods: Prospective, non-randomized, single-center clinical study that included 9 CXLtreated keratoconus eyes followed with corneal topography, specular microscopy and AS-OCT for 12 months.

Results: Both reduction of keratometric values and corneal thinning were evidenced in CXLtreated eyes, whereas untreated fellow eyes showed topographic progression. Three structural changes were found with AS-OCT: increase of anterior $(180 \pm 20 \mu \mathrm{m})$ stroma reflectivity that peaked at six months; hyperreflective demarcation line in posterior stroma (365 $\pm 56 \mu \mathrm{m})$ that gradually reduced its intensity until becoming undetectable in $50 \%$ of the cases after 12 months; and incomplete secondary hyperreflective lines located at variable depths that gradually disappeared.

Conclusions: AS-OCT is a useful tool for assessment of CXL results since it can detect quantitative and qualitative modifications induced by this treatment.

Categories: Ophthalmology

Keywords: corneal crosslinking, keratoconus, optical coherence tomography, anterior segment optical coherence tomography

Published 10/15/2012

๑) Copyright 2012

Fuentes Bonthoux et al. This is an open access article distributed under the terms of the Creative Commons Attribution License CCBY 3.0., which permits unrestricted use, distribution, and reproduction in any medium, provided the original author and source are credited.

\section{Introduction}

Keratoconus is a non-inflammatory corneal ectasia characterized by progressive thinning of the stroma. Since a lower number of interfibrillar unions in collagen lamellae have been linked to its pathogenesis [1], corneal crosslinking (CXL) induction with riboflavin and ultraviolet A (UVA) light constitutes an attractive treatment [2]. Beneficial effects of CXL aside, UVA radiation can induce the apoptosis of endothelial cells if sufficiently exposed [3], and thus, its penetration depth must be controlled. Recently, a demarcation line was biomicroscopically 
described that would represent the transition area between treated and untreated stroma [4] at an approximate depth of $300 \mathrm{gm}$. Evidently CXL induces microscopic modifications that benefit the overall corneal structure. Anterior segment optical coherence tomography (AS-OCT) can image the corneal stroma with high resolution, and therefore the goal of this prospective study was to describe its findings in CXL-treated patients.

\section{Materials And Methods}

The study was a prospective, non-randomized clinical study. The research protocol followed the tenets of the Declaration of Helsinki and was approved by the Hospital de Clinicas Ethics Committee. All subjects were told of the purpose of the study and gave written informed consent before inclusion. Patients were recruited at the Cornea Section of the Ophthalmology Division of the hospital, and the study was completed between October 2005 and April 2008. Only the eye with the more advanced disease (defined by the highest average corneal power) was treated if it showed evolutive changes (increase in the contact lens base curvature or steepest SimK change > 1D in one year), had central corneal thickness greater than $400 \mathrm{gm}$, no corneal opacities, no other eye disease, nor previous surgery. After 14 days without wearing contact lenses (and repeated every three months after treatment), patients underwent endothelial cell count (TopCon SP-2000P, TopCon America, Paramus, NJ, USA), topography (KernTech 2000, Pfortner Laboratory, Buenos Aires, Argentina), and AS-OCT (Visante OCT, Carl Zeiss Meditec, Dublin, CA, USA) with polarization set to 50\%. Statistical analysis was performed with Prism 5 software (GraphPad Software, La Jolla, CA, USA). Continuous variables are expressed as mean \pm SD. Statistical significance was set at $\mathrm{p}<0.05$.

\section{Surgical technique}

The surgical procedure started with antisepsis, topical anesthesia, corneal epithelium removal under a surgical microscope, instillation of riboflavin $0.1 \%$ (in dextran $20 \%$ solution) every three minutes for 30 minutes prior to and during irradiation. The 370 -nm UVA radiation source (CLII TM device, Pentium Laboratory, Buenos Aires, Argentina) was then focused on the corneal apex for 30 minutes, delivering a measured irradiance of $3 \mathrm{~mW} / \mathrm{cm}^{2}$ over the central $7 \mathrm{~mm}$. A soft contact lens was applied after the eye was washed with balanced solution. Postoperatively, patients received gatifloxacin four times/day and ketorolac $0.5 \%$ and prednisolone acetate 1\%/phenylephrine hydrochloride every 12 hours. After re-epithelization was achieved in all cases within seven days, only fluorometholone was indicated three times daily. For three months, patients were prescribed oral supplements of ascorbic acid, vitamin B complex, and amino acids.

\section{Results}

The studied group comprised four women and five men (age: 29 \pm , range 19-49 years) who attended every follow-up visit. Keratoconus severity among the five right eyes and four left eyes treated was two Grade I cases, three Grade II cases and four Grade III cases. Evolution of corneal pachymetry, topography and endothelial cell count is shown in Table 1.

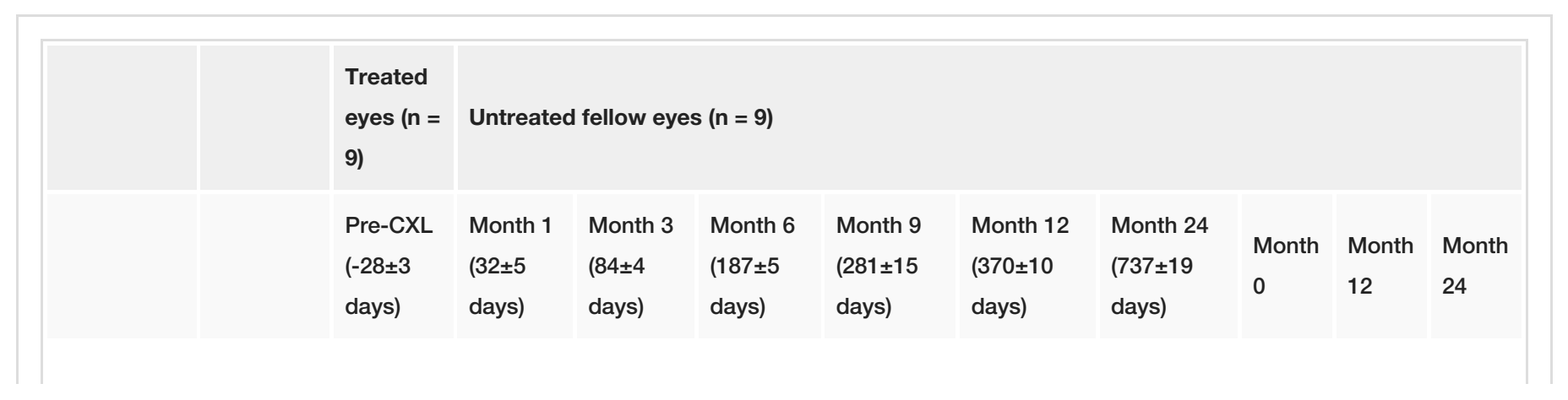




\section{Cureus}

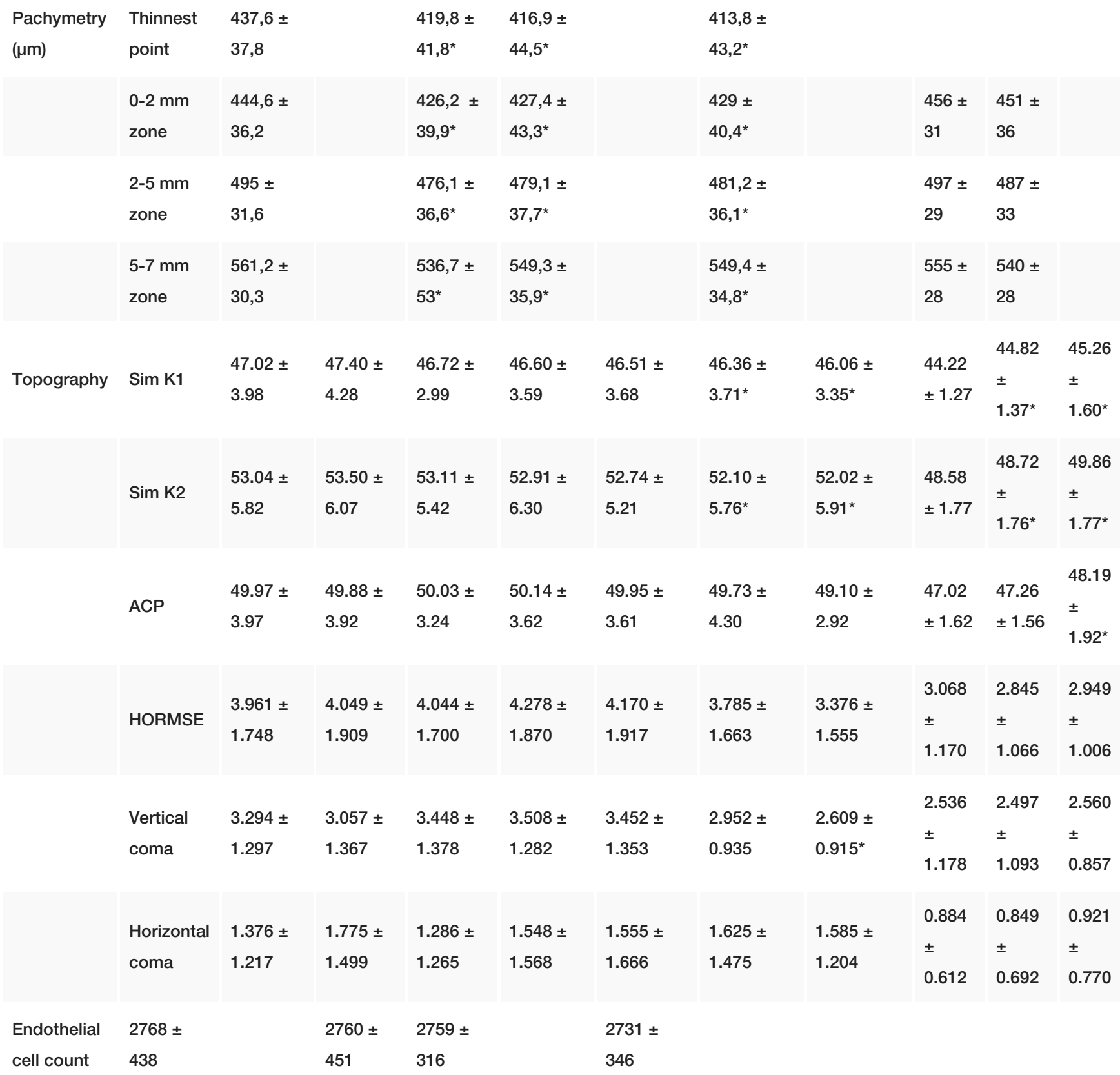

\section{TABLE 1: Pachymetric, topographic and endothelial evolution of CXL-treated and untreated fellow eyes}

Data expressed as mean \pm SD. * Statistically significant difference compared to pre-CXL measurement $(p<0.05$, Wilcoxon's matched pairs test).

Three qualitative changes were observed with AS-OCT in the CXL-treated corneae: increase in anterior stroma reflectivity, appearance of a demarcation line and of unexpected secondary lines (Figure 1). The reflectivity increase was limited to the anterior $180 \pm 20 \mu \mathrm{m}$, with the following time pattern: after three months, three patients presented a distinguishable but subtle increase, in five patients this increase was easily visible and in one patient it was marked. After six months reflectivity intensified, but after one year it returned to three-month levels. The appearance of a sharp hyperreflective demarcation line in the posterior stroma was 


\section{Cureus}

observed in all treated eyes. After three months, the average depth was $365 \pm 46 \mu \mathrm{m}$. A significant attenuation of its intensity was observed after six months, remaining identifiable only in four eyes after one year. Lesser hyperreflective, incomplete lines were observed at variable levels and were called secondary lines. During the first six months, they were identifiable in six cases and then faded away, remaining visible in only one eye after one year (Figure 1).
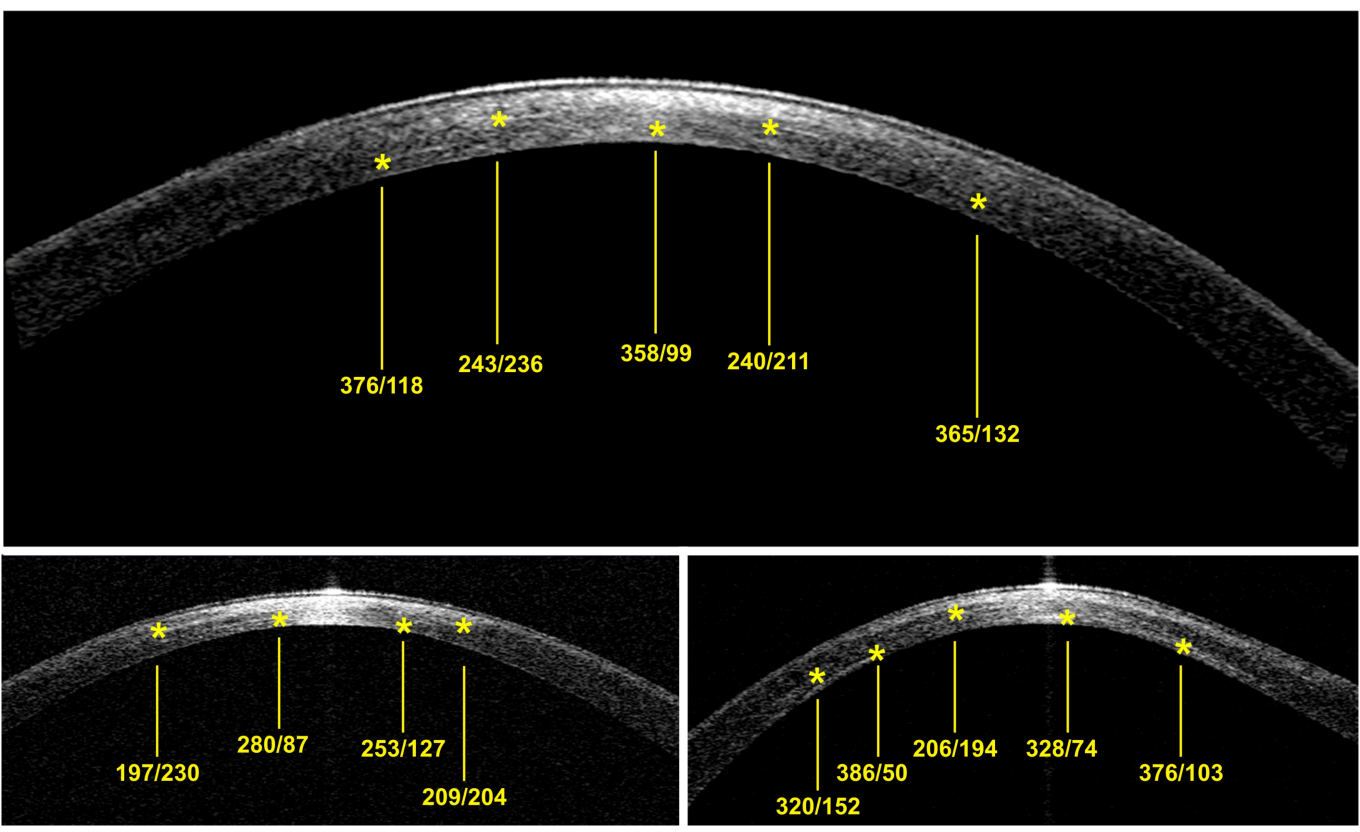

\section{FIGURE 1: Representative examples from three patients of corneal high-resolution OCT images of the horizontal meridian at 6 months post-CXL.}

* indicate hyperreflective stromal lines with depth / posterior strom

\section{Discussion}

Several studies have demonstrated the effectiveness and safety of CXL to stop the evolution of keratoconus [5-6]. Accordingly, there was a comparable halt in keratoconus progression in our series with no impact on the endothelial cell count. In comparison, the untreated fellow eyes had higher keratometric values after 12 months, reflecting the evolutive nature of the disease. Additionally, we observed a reduction in corneal thickness which was greater at the three month follow-up and remained stable after one year. Others have previously reported comparable thinning with Scheimpflug imaging [7] and with AS-OCT [8]. It remains to be established whether these findings reflect true corneal thinning or are artifacts caused by changes in the optical properties of the corneal stroma after CXL.

As pointed out earlier, CXL treatment must be performed under an exquisite balance between riboflavin concentration and UVA radiation, and the reported ideal combination provides a maximum treatment activity in the anterior 300 lam of the cornea with almost no effect at deeper levels [3]. A recent study with AS - OCT [8] identified the stromal demarcation line that allegedly represents the treatment penetration depth [4, 9] and further explored its implications. In our series, we observed this finding in all patients at three and six months as a hyperreflective line at an average depth of $365 \pm 56$ gm that progressively reduced its intensity until becoming unidentifiable in $50 \%$ of the cases after 12 months. We 'detected two additional 
changes in the high-resolution corneal images: an increase in the anterior stroma reflectivity and the appearance of secondary lines (Figure 1).

In all patients, we observed an increase in the anterior stroma reflectivity that remained readily noticeable after 12 months. This finding might correlate with the confocal microscopy observations of increase in the extracellular matrix reflectivity and keratocyte hyperplasia in the anterior stroma [9-10]. Surprisingly, some patients also showed incomplete secondary hyperreflective lines at variable depths, which due to their parallel disposition to stromal lamellae and progressive attenuation might reflect local differences in treatment effect within the stroma. To the best of our knowledge, these complementary lines have not been reported before; we believe they represent incomplete demarcation lines that appear at different depths because of fluctuation in corneal thickness during the irradiation period (both isotonic and hypertonic riboflavin solutions are used complementarily to compensate for these pachymetric changes).

\section{Conclusions}

All together, these three AS-OCT signs could be used to monitor the effective treatment depth in clinical practice in a more objective and reproducible manner than slit-lamp biomicroscopy. In this regard, Doors, et al. found a positive correlation between the demarcation line depth and corneal thickness reduction [8]. To sum up, AS-OCT can consistently identify qualitative changes in corneae treated with CXL. The clinical meaning of these findings and their possible predictive value remain to be identified.

\section{Additional Information \\ Disclosures}

Human subjects: Consent was obtained by all participants in this study. The Hospital de Clinicas Ethics Committee issued approval \# 20050905/1. The research protocol followed the tenets of the Declaration of Helsinki and was approved by the Hospital de Clinicas Ethics Committee. All subjects were told of the purpose of the study and gave written informed consent before inclusion. Animal subjects: All authors have confirmed that this study did not involve animal subjects or tissue. Conflicts of interest: In compliance with the ICMJE uniform disclosure form, all authors declare the following: Payment/services info: All authors have declared that no financial support was received from any organization for the submitted work. Financial relationships: All authors have declared that they have no financial relationships at present or within the previous three years with any organizations that might have an interest in the submitted work. Other relationships: All authors have declared that there are no other relationships or activities that could appear to have influenced the submitted work.

\section{References}

1. Daxer A, Fratzl P: Collagen fibril orientation in the human corneal stroma and its implication in keratoconus.. Invest Ophthalmol Vis Sci. 1997, 38:121-129.

2. Spoerl E, Huhle M, Seiler T: Induction of cross-links in corneal tissue . Exp Eye Res. 1998, 66:97-103.

3. Wollensak G, Spoerl E, Reber F, Seiler T: Keratocyte cytotoxicity of riboflavin/UVA-treatment in vitro. Eye . 2004, 18:718-722.

4. Seiler T, Hafezi F: Corneal cross-linking-induced stromal demarcation line . Cornea. 2006, 25:1057-1059.

5. Raiskup-Wolf F, Hoyer A, Spoerl E, Pillunat LE: Collagen crosslinking with riboflavin and ultraviolet-A light in keratoconus: Long-term results. J Cataract Refract Surg. 2008, 34:796801. 10.1016/j.jcrs.2007.12.039

6. Caporossi A, Mazzotta C, Baiocchi S, Caporossi T: Long-term results of riboflavin ultraviolet a 


\section{Cureus}

corneal collagen cross-linking for keratoconus in Italy: The Siena eye cross study. Am J Ophthalmol. 2010, 149:585-593. 10.1016/j.ajo.2009.10.021

7. Vinciguerra P, Albè E, Trazza S, Rosetta P, Vinciguerra R, Seiler T, Epstein D: Refractive, topographic, tomographic, and aberrometric analysis of keratoconic eyes undergoing corneal cross-linking. Ophthalmology. 2009, 116:369-378. 10.1016/j.ophtha.2008.09.048

8. Doors M, Tahzib NG, Eggink FA, Berendschot TT, Webers CA, Nuijts RM: Use of anterior segment optical coherence tomography to study corneal changes after collagen cross-linking. Am J Ophthalmol. 2009, 148:844-851. 10.1016/j.ajo.2009.06.031

9. Mazzotta C, Bales A, Traversi C, Baiocchi S, Caporossi T, Tommasi C: Treatment of progessive keratoconus by riboflavin-OVA-induced cross-linking of corneal collagen: Ultrastructural analysis by Heidelberg Retinal Tomogaph II in vivo confocal microscopy in humans. Cornea . 2007, 26:390-397.

10. Mazzotta C, Traversi C, Baiocchi S, Caporossi O, Bovone C, Sparano MC, Balestrazzi A, Caporossi A: Corneal healing after riboflavin ultraviolet-A collagen cross-linking determined by confocal laser scanning microscopy in vivo: early and late modifications. Am J Ophthalmol. 2008, 146:527-533. 10.1016/j.ajo.2008.05.042 